\title{
Detection of neutral sodium above Mercury during the transit on 2003 May 7
}

\author{
H. Schleicher ${ }^{1}$, G. Wiedemann ${ }^{2}$, H. Wöhl ${ }^{1}$, T. Berkefeld ${ }^{1}$, and D. Soltau ${ }^{1}$ \\ 1 Kiepenheuer-Institut für Sonnenphysik, Schöneckstr. 6, 79104 Freiburg, Germany \\ e-mail: schleicher@kis.uni-freiburg.de \\ ${ }^{2}$ Hamburger Sternwarte, Gojenbergsweg 112, 21029 Hamburg, Germany
}

Received 19 March 2004 / Accepted 1 June 2004

\begin{abstract}
The extent of the exosphere of Mercury above the planet's limb could for the first time be observed by detecting an excess absorption in the solar sodium line $\mathrm{D}_{2}$ during the transit of Mercury across the solar disk on 2003 May 7. The observations were performed with a 2d Fabry-Perot spectrograph of the Vacuum Tower Telescope at Izaña, Tenerife. The absorption excess, blue-shifted by $13 \mathrm{pm}$ relative to the solar line, is mainly concentrated near the polar regions. There, the absorption excess can be traced up to $\approx 700 \mathrm{~km}$ above the limb. Between the two polar regions, along the eastern limb, a weaker absorption excess can be seen. A possible streamer-like feature stretches more than $2000 \mathrm{~km}$ above the northern region. Assuming the density to decrease exponentially with height, we derive for the polar maxima vertical column densities of $3 \times 10^{10} \mathrm{~cm}^{-2}$, volume densities at the surface of $2.5 \times 10^{3} \mathrm{~cm}^{-3}$, and a density scale height of $150 \mathrm{~km}$.
\end{abstract}

Key words. planets and satellites: individual: Mercury

\section{Introduction}

The existence of Mercury's exosphere has been known since the fly-by of Mariner 10 (Bradfood et al. 1976). It was nevertheless a surprise that Potter \& Morgan (1985) detected narrow emission lines at the sun-illuminated disk, superimposed on the solar absorption resonance lines of sodium. In the meantime, the existence of such emission has been established by several other observations, not only in the sodium lines, but also in the resonance lines of potassium (Potter \& Morgan 1986) and calcium (Bida et al. 2000).

The line emission is seen on the illuminated part of the planet's disk. Although ground-based observations are strongly hampered by seeing effects, there are indications that the emission varies in time and is not uniformly distributed over the surface, with a preference of being concentrated near the polar regions (Potter et al. 1999). Killen et al. (1990) derived sodium atom column densities of several $10^{11} \mathrm{~cm}^{-2}$. The atoms (other than $\mathrm{H}, \mathrm{He}, \mathrm{O}$ ) forming the exosphere are sputtered from the planet's surface by solar wind particles and/or by solar EUV photons. An additional release mechanism might be bombardment by meteorites. After ionization by the solar UV radiation, the ions interact with Mercury's magnetic field which might explain the inhomogeneous nature of the exosphere. For models of the exosphere see, e.g., Ip (1993), or Lammer et al. (2003).

In the above cited observations, the line emission is seen projected on the illuminated part of the planet's disk. Here, we report the first successful detection of absorption above the limb of Mercury due to the planet's exosphere in the sodium resonance line $\mathrm{D}_{2}(\lambda 589.0 \mathrm{~nm})$ on occasion of the transit on 2003 May 7.

\section{Observation and data reduction}

We observed the transit of Mercury across the solar disk on 2003 May 7 with the Vacuum Tower Telescope VTT (Schröter et al. 1985) at the Observatorio del Teide in Izaña at Tenerife. Using the two-dimensional Fabry-Perot spectrograph TESOS (Kentischer et al. 1998; Tritschler et al. 2002) we scanned the central part of the $\mathrm{Na}_{2}$ line, with a spectral resolution of $2 \mathrm{pm}$ and oversampling by a factor 2.2. Because of the telecentric mounting of the etalons, the wavelength of transmission maximum does not significantly vary over the field-of-view; residual effects are suppressed when applying the standard CCD flatfield correction. Image degradation due to seeing was compensated by the adaptive optics system KAOS (Soltau et al. 2002). Observational parameters are given in Table 1.

The sky was cloudless, the seeing varied between $2^{\prime \prime}$ and $1^{\prime \prime}$. Thanks to the AO-system, the residual seeing effects were $\leq 0.5^{\prime \prime}$, except for instances of very bad seeing. The observations of the transit started at 8 UT when the sun was $20^{\circ}$ above horizon, $10 \mathrm{~min}$ after the planet had crossed the solar meridian. Since the transit campaign was devoted to several topics, scans of the $\mathrm{Na} \mathrm{D}_{2}$ line were done for somewhat less than $1 \mathrm{~h}$ during the final two hours of the transit, yielding 
Table 1. Observational parameters.

\begin{tabular}{l|c|l}
\hline \hline Time of Na D session & UT & start 8:18 end 10:33 \\
Time of selected scans & UT & first 8:27 last 10:25 \\
Central wavelength & $\mathrm{nm}$ & $589.0\left(\mathrm{Na} \mathrm{D}_{2}\right)$ \\
Scan range & $\mathrm{pm}$ & -49.9 to +30.8 \\
Scan steps & $\mathrm{pm}$ & 0.89 \\
Number of steps /scan & & 92 \\
(=number of frames) & & \\
Spectral resolution $(F W H M)$ & $\mathrm{pm}$ & 2 \\
Frame exposure time & $\mathrm{ms}$ & 200 \\
Scan cadence time & $\mathrm{s}$ & 32 \\
Total number of scans & & 130 \\
Number selected scans & & 22 \\
Field of view & $\operatorname{arcsec}$ & 42 \\
Image pixel size & $\operatorname{arcsec}$ & 0.09 \\
\hline
\end{tabular}

130 scans in total. However, in many scans Mercury was near or even outside the field-of-view boundary of the TESOS spectrograph because the planet had intentionally been offset several times in order to test the performance of the AO system KAOS.

For the search of an absorption excess, the best 22 scans were selected according to the following criteria:

- planet center $>12^{\prime \prime}$ (one disk diameter) away from field-of-view boundary;

- pointing stability during the scan $<1^{\prime \prime}$;

- contrast of solar granules above a sufficient threshold.

Each scan consists of a set of 92 filter images ("frames") with 2 pm spectral resolution, the central wavelength increasing from frame to frame by $0.89 \mathrm{pm}$ because of the step-wise incrementation of the plate separations of the three Fabry-Perot etalons. Hence, from the set of 92 frames, the spectral information (here: the central $0.08 \mathrm{~nm}$ of the $\mathrm{D}_{2}$ line) can be obtained for each pixel inside the field-of-view.

For each frame of a scan (hence, for each wavelength step) intensity counts are extracted according to the following steps:

- Determination of the disk center (accuracy \pm 0.2 pix $\left.\left( \pm 0.018^{\prime \prime}\right)\right)$;

- pixel-step sampling of counts along concentric circles with radii increasing from $5.2^{\prime \prime}$ to $10.8^{\prime \prime}$, corresponding to distances of $-321 \mathrm{~km}$ to $+1961 \mathrm{~km}$ away from Mercury's limb (the planet's disk had an apparent radius of $6.0^{\prime \prime}$ );

- averaging over $15^{\circ}$ azimuthal bins to suppress noise.

In total, "local" profiles of the $\mathrm{Na}_{2}$ line for 65 radial distances $r$ and for 24 azimuthal directions $\varphi$ are obtained for each scan. For each scan we construct a "reference profile" by averaging the profiles over the outmost 16 sampled circles ( $>9.5^{\prime \prime}$ off the planetary limb) and all azimuthal directions. Comparison of line profiles from the near vicinity of Mercury's disk with the reference will reveal any additional absorption produced by Mercury's exosphere. During the transit the radial velocity of Mercury relative to the Earth was $-4.6 \mathrm{~km} \mathrm{~s}^{-1}$, while the radial velocity of the Sun (including gravitational redshift) increased from $+1.40 \mathrm{~km} \mathrm{~s}^{-1}$ to $+2.29 \mathrm{~km} \mathrm{~s}^{-1}$ because of the increasing influence of the solar rotation when Mercury was moving towards the western solar limb. The additional absorption due to the exosphere, if present, should therefore show up as a depression in the solar $\mathrm{Na}_{2}$ profile, blue-shifted by $12.1-13.5 \mathrm{pm}$ relative to the center of the the solar line. Figure 1 shows a typical local line profile together with the reference. The absorption excess at distance $r$ and azimuth $\varphi$ is determined, after applying several corrections, as the relative intensity difference between the reference profile $I_{\text {ref }}(\lambda)$ and local profile $I(\lambda, r, \varphi)$ according to

$\Delta I(\lambda, r, \varphi)=\left(I_{\text {ref }}(\lambda+\delta \lambda)-I(\lambda, r, \varphi) f(r, \varphi)\right) / I_{\text {ref }}(\lambda+\delta \lambda)$

with

$f(r, \varphi)=\left\langle I_{\mathrm{ref}}\right\rangle_{\lambda} /\langle I(\lambda, r, \varphi)\rangle_{\lambda}$.

The factor $f(r, \varphi)$, derived from matching the wing intensities of the two profiles, corrects the diminution of light near the planet's disk due to scattering (the missing light is scattered onto the disk). The "Doppler compensation" $\delta \lambda$ is applied to correct small wavelength shifts due to large-scale solar velocity variability and is determined by matching the very line cores of the profiles. As can be seen in Fig. 1, the extracted profiles exhibit a periodic intensity modulation in wavelength. This effect is caused by an imperfect plate of one of the three etalons of the spectrograph. The modulation nearly cancels when taking the difference between the local and reference profile; nevertheless, we cleaned all profiles by applying a suitable Fourier filter.

Finally, the equivalent widths $W_{\lambda}(r, \varphi)$ are computed by integrating the relative intensity differences over 14 wavelength steps around the expected center of the Mercury absorption line and apply a "core-width correction":

$$
\begin{aligned}
& W_{\lambda}(r, \varphi)=\int_{\lambda_{0}^{M} \pm \Delta \lambda} \Delta I(\lambda, r, \varphi) \mathrm{d} \lambda-\text { c.w.c. with } \\
& \text { c.w.c. }=\int_{\lambda_{0}^{*} \pm \Delta \lambda} \Delta I(\lambda, r, \varphi) \mathrm{d} \lambda, \quad \lambda_{0}^{*}=\lambda_{0}^{\odot}+\left(\lambda_{0}^{\odot}-\lambda_{0}^{M}\right)
\end{aligned}
$$

$\lambda_{0}^{M}$ and $\lambda_{0}^{\odot}$ are the predicted line center wavelengths of the Mercury absorption line and the solar line. The core-width correction c.w.c. accounts for spatial variability of the width of the line cores and is estimated by integrating $\Delta I(\lambda, r, \varphi)$ in the red line core over the wavelength interval of the Mercury feature, mirrored around the center of the solar profile. This correction assumes that the cores of the solar $\mathrm{Na}_{2}$ line profiles are symmetric. It should be mentioned that the "Doppler" and "core-width correction" are not essential regarding the qualitative results. Applying these corrections reduces significantly the noise of the data, however. The achieved accuracy of $W_{\lambda}(r, \varphi)$ is mainly limited by the spatial variability of the solar line profile. The local values obtained in areas not influenced by Mercury are scattering around zero by $\approx 0.3 \mathrm{pm} \mathrm{rms}$ for single scans; the rms of the median over the 22 selected scans is $\approx 0.07 \mathrm{pm}$, with no systematic variation along the azimuth. Figure 2 shows the difference between the local profile shown in Fig. 1 and its reference, normalized by the latter. Although the noise is substantial, the existence of an absorption excess at the predicted wavelength is evident (brown area). 


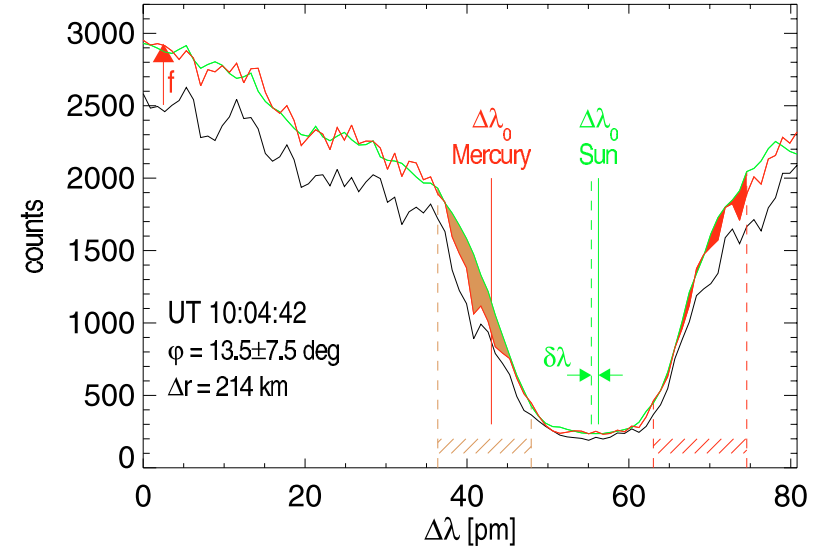

Fig. 1. Profile of $\mathrm{Na}_{2}$ extracted $214 \mathrm{~km}$ above Mercury's limb in the northern polar region. Black: original local profile, green: Doppler compensated reference profile, red: local profile, multiplied by a factor $f$ (see Eq. (2)) to match the wing intensities of the reference. The red and green profile have been cleaned as described in the text. The vertical red line marks the predicted location of the line center of the Mercury absorption feature, the vertical green line is the predicted location of the line center of the solar line. The green dashed line indicates the Doppler shift $\delta \lambda$ between local and reference profile. The excess absorption due to Mercury's exosphere is the difference between the green and red profile around the expected central wavelength and is emphasized by the brown area. The red area in the red line wing is the "core-width correction" (see text). The vertical dashed brown and red lines mark the integration boundaries used to retrieve the equivalent width of the absorption excess and the corewidth correction.

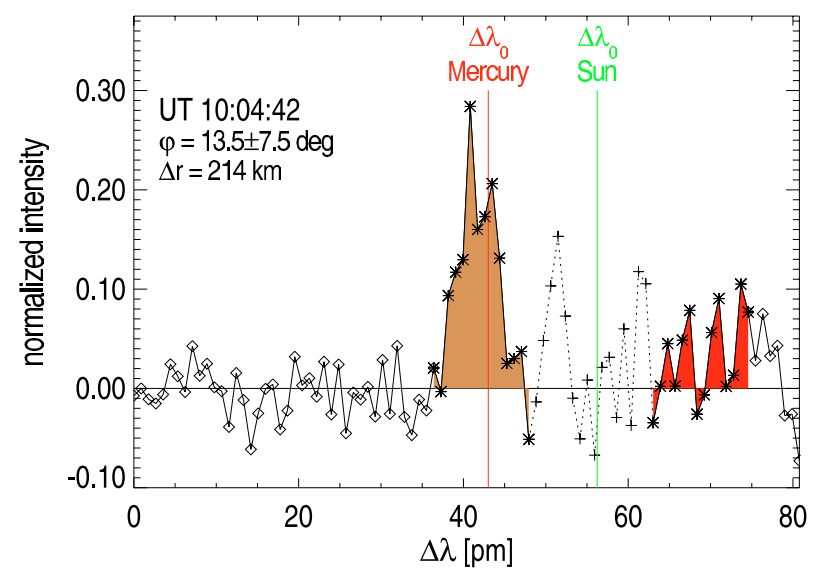

Fig. 2. Difference between the local and reference profile shown in Fig. 1. The counts have been normalized by the counts of the reference. Positive values indicate excess of absorption. The brown area is proportional to the uncorrected equivalent width $\tilde{W}_{\lambda}$ of the absorption excess. The red area will be subtracted from $\tilde{W}_{\lambda}$ to correct for spatial variability of the width of the solar line ("core-width correction", see text). Around the center of the solar line, the values are very noisy due to the low intensity level (dashed part of the difference profile).

We can exclude several possible effects that might produce artificial line depression of the kind seen in Figs. 1-3:

- An absorption blend of solar or telluric origin, if present at the wavelengths in question, would be compensated for by the subtraction of the local profile from the reference.

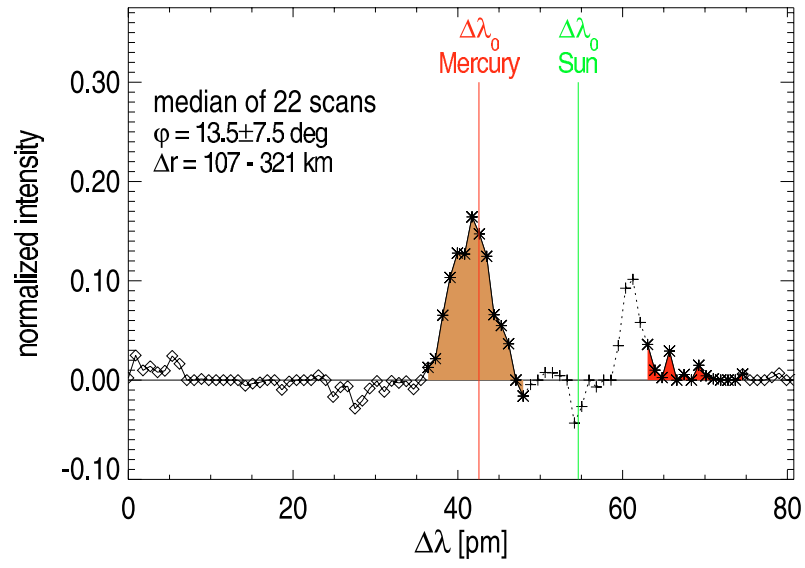

Fig. 3. Same as Fig. 2, difference-profile averaged in radial direction over $214 \mathrm{~km}\left(0.5^{\prime \prime}\right)$ and median averaged over the 22 selected scans.

- Seeing influences produce scatter randomly distributed along the profiles; of course, line profiles obtained near Mercury's limb are more affected than the reference. For an individual profile scan, seeing might produce an artificial distortion affecting several wavelength steps in sequence. However, it is very unlikely that for many scans always the same wavelength points are affected in the same manner. Seeing is, however, an important source of the data noise.

- Differences between local and reference profile of solar origin (center-to-limb variation, large-scale velocity field and temperature inhomogeneities of the chromosphere) are compensated as described above (correction terms $\delta \lambda$, and c.w.c., Eqs. (2), (4)); even when these corrections are neglected, the results do not change qualitatively.

- Systematic variation of the general intensity level has been corrected by the position dependent factor $f(r, \varphi)$, Eq. (2). This factor, determined in the line wings, includes effects of scattered light (main effect) and gradients over the field-ofview due to the solar center-to-limb variation, the latter is noticeable only for the very last scans before the 3rd contact. Even if not been corrected properly, the whole line profile would be affected, not just a few wavelength steps, and large-scale variations over the field-of-view should be seen, which is not the case.

\section{Results}

Figures 2 and 3 show the profile differences at a location $\approx 215 \mathrm{~km}$ above the northern planetary limb. Even for a single scan and no radial averaging (Fig. 2) the signal for an excess absorption at the predicted wavelengths is high $(\sigma \approx 7)$. Since we find an additional line depression of comparable strength only near Mercury's limb, we are confident that this depression must be due to excess absorption by the exosphere of the planet. In Fig. 3, where we have averaged the profile differences over $0.5^{\prime \prime}$ (about the achieved spatial resolution) and over all selected scans, some non-significant deviations from zero are seen around the center of the solar profile. We ascribe these deviations to an artifact produced by a small over-estimation of the "Doppler compensation" (see Chap. 2). One must be 

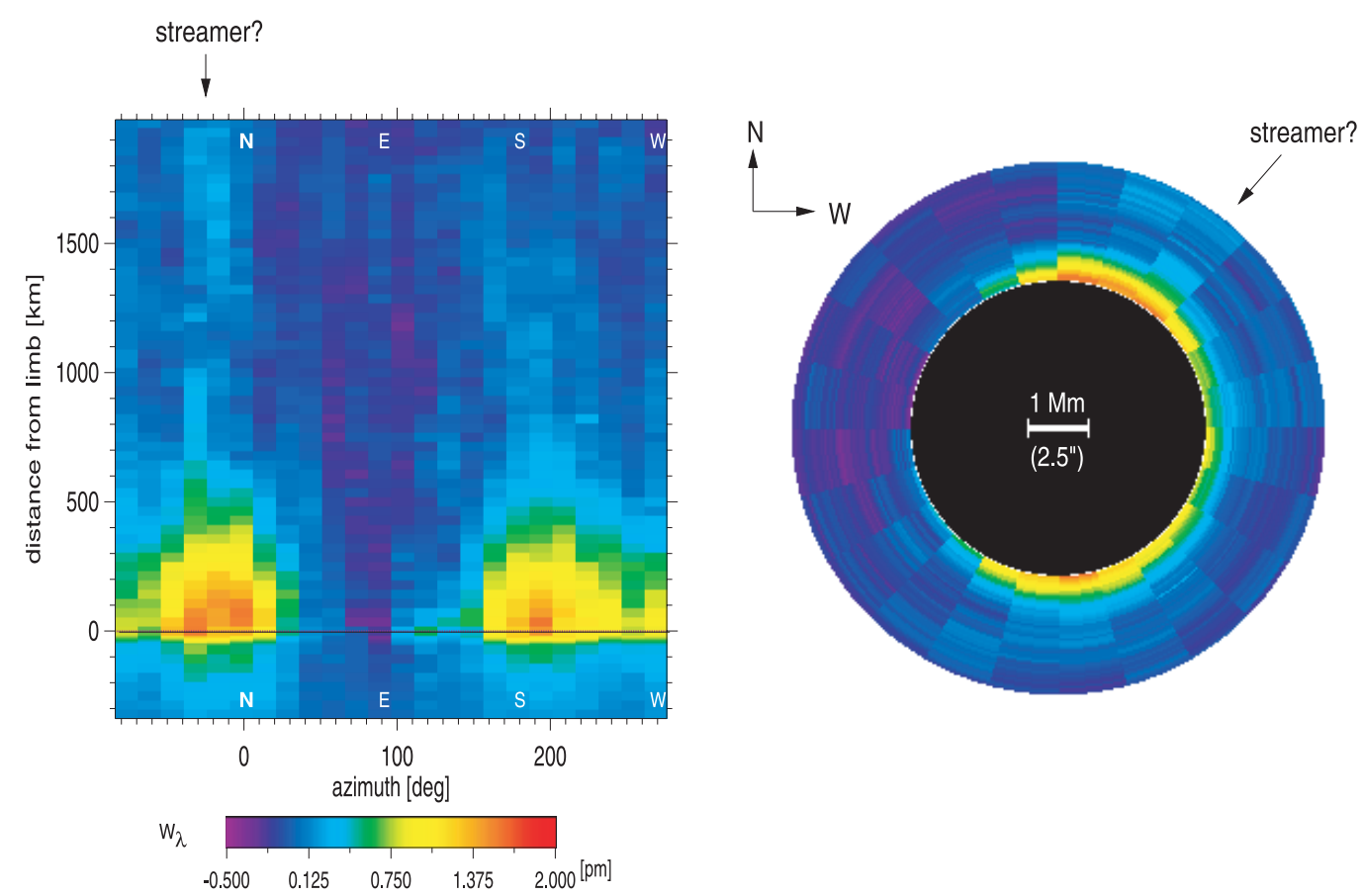

Fig. 4. Distribution of observed equivalent widths $W_{\lambda}$ above Mercury's limb. $\Delta r$ is the distance from the planetary limb. Inside the disk $(\Delta r<0)$, the equivalent widths are multiplied with the relative intensity distribution of the scattered light.

aware, that any small deviation of local and reference profile near the solar line center is magnified strongly by the normalization with the reference profile and due to the fact that the solar $\mathrm{D}_{2}$ line is very dark in its center. In Fig. 4 the spatial distribution of the equivalent widths $W_{\lambda}(\varphi, r)$ of the absorption excess is shown. Here, and for all further discussion, the data are median values of the 22 selected scans. The zero-point of the azimuthal variable $\varphi$ is the direction of the normal to Mercury's orbital plane which nearly coincides with the direction of the rotation axis. We could determine the orientation of the orbital plane in the spectrograph images to within $1^{\circ}$. The results, as derived directly from the observations, can be summarized as follows:

1. Above both polar regions an absorption excess of high significance is seen. The maxima of excess absorption occur at azimuth angles $16^{\circ}$ and $14^{\circ}$ west of the north and south direction, rsp.

2. The maximal equivalent width $W_{\lambda}$ at both polar regions of $\approx 1.5 \mathrm{pm}$ occurs immediately at the planetary limb. The southern absorption can be traced up to $700 \mathrm{~km}$ above the limb, where $W_{\lambda}$ approaches the detection limit of $\approx 0.1 \mathrm{pm}$.

3. At both polar regions the absorption is concentrated within two azimuthal segments of $45^{\circ}(1900 \mathrm{~km})$ along the limb.

4. No excess absorption above the detection limit is found along the eastern planetary limb. A weak absorption with $W_{\lambda} \approx 0.3 \mathrm{pm}$ can be detected along the western limb, connecting the two polar regions.

5. Above the northern maximum, at azimuth $25^{\circ} \mathrm{W}$, a streame-like excess absorption with $W_{\lambda} \approx 0.15 \mathrm{pm}$ is found which can be traced in radial direction to the boundary of measurements, $1900 \mathrm{~km}$ above Mercury's limb. Since the strength of absorption is near the detection limit, the reality of this feature is questionable, however.

6. The wavelength dependence of the excess absorption (see Fig. 3) can be approximated by a Gaussian profile $\sim \exp \left(-\left(\Delta \lambda / \Delta \lambda_{\mathrm{D}}\right)^{2}\right)$ with $\Delta \lambda_{\mathrm{D}}=3.3 \mathrm{pm}$. Taking into account the spectral resolution of the spectrograph, the intrinsic Doppler width is $3.1 \pm 0.1 \mathrm{pm}$, corresponding to a velocity distribution along the line-of-sight of $1.6 \pm 0.1 \mathrm{~km} \mathrm{~s}^{-1}$. No significant spatial variation of the line widths is found.

In order to derive the density distribution of the sodium atoms, the influence of light scattering on the observed distribution of the equivalent widths must be taken into account. We proceed as follows:

In the vicinity of the polar maxima the density distribution $n(r, \varphi)$ of the neutral sodium atoms in the ground state at distance $r$ from Mercury's center and azimuthal angle $\varphi$ is modeled by ( $R$ the radius of Mercury)

$n(r, \varphi)=n_{0} \phi(\varphi) \exp \left(-\frac{r-R}{H}\right) \quad(r \geq R)$
$\phi(\varphi)=\exp \left(-\frac{\varphi-\varphi_{\max }}{\Delta \varphi}\right), \quad \Delta \varphi=30^{\circ}$.

The line absorption coefficient per volume:

$\kappa_{\Delta \lambda}=n(r, \varphi) \kappa_{0}^{(A)} \frac{1}{\sqrt{\pi} \Delta \lambda_{\mathrm{D}}} \exp \left(-\left(\frac{\Delta \lambda}{\Delta \lambda_{\mathrm{D}}}\right)^{2}\right)$,

$\kappa_{0}^{(A)}=\frac{\lambda_{0}^{4}}{8 \pi c} \frac{g_{i}}{g_{j}} A_{j i}$

$\left(\mathrm{Na} \mathrm{D}_{2}: \lambda_{0}=0.859 \mu, g_{i}=2, g_{j}=4, A_{j i}=6.30 \times 10^{7} \mathrm{~s}^{-1}\right)$ 


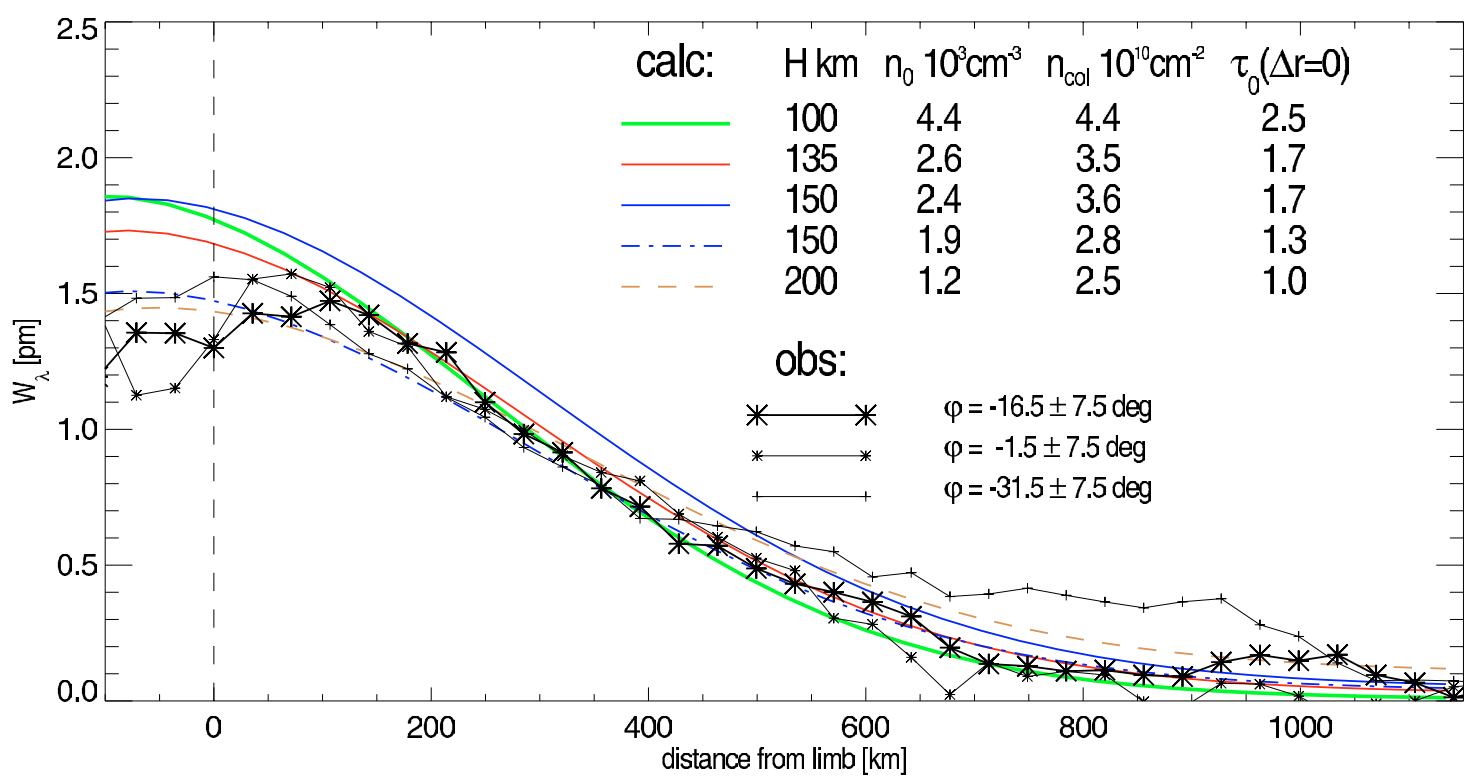

Fig. 5. Height dependence of equivalent widths. The black, marked curves are values observed above the northern pole. The non-vanishing values for $\varphi=-31.5^{\circ}$ and $\Delta r>700 \mathrm{~km}$ can be ascribed to the "streamer" feature. The unmarked curves have been calculated, as described in the text. The used combinations of scale height $H$ and particle density $n_{0}(\Delta r=0)$ are given in the legend, together with the resulting column densities $n_{\text {col }}$ and line center optical depths along the grazing line-of-sight, $\Delta \tau_{0}(\Delta r=0)$. The red curve is the best fit for $\varphi=-16.6^{\circ}$, see also Table 2.

Table 2. Results of spectral analysis.

\begin{tabular}{l|lllll}
\hline \hline Location & $\varphi$ & $\tau_{0}$ & $H$ & $n_{0}$ & $n_{\text {col }}$ \\
\hline & $\mathrm{deg}$ & & $\mathrm{km}$ & $10^{3} \mathrm{~cm}^{-3}$ & $10^{10} \mathrm{~cm}^{-2}$ \\
\hline Northern maximum & -16. & $1.7 \pm \operatorname{dex} 0.15$ & $135 \pm 30$ & $2.6 \pm \operatorname{dex} 0.2$ & $3.4 \pm \operatorname{dex} 0.1$ \\
Southern maximum & 193.5 & $1.5 \pm \operatorname{dex} 0.15$ & $130 \pm 30$ & $2.3 \pm \operatorname{dex} 0.2$ & $3.0 \pm \operatorname{dex} 0.1$ \\
Western planetary limb & -91.5 & $0.7 \pm 0.1$ & $(150)$ & $1.0 \pm \operatorname{dex} 0.2$ & $1.5 \pm \operatorname{dex} 0.1$ \\
Eastern planetary limb & 73.5 & $<0.1$ & $(150)$ & $<0.14$ & $<0.2$ \\
\hline$\varphi$ : azimuth angle measured from north pole towards east. & & \\
$\tau_{0}$ : optical depth at line center, along grazing line-of-sight $(\Delta r=0)$. & & \\
$H:$ density scale height, Eq. (4); E-,W-limb: values assumed for calculation. & \\
$n_{0}$ : density at surface of neutral sodium atoms at ground level (Eq. (4)). & \\
$n_{\text {col } \text { : column density of neutral sodium atoms at ground level. }}$ &
\end{tabular}

is integrated along each line-of-sight to obtain the optical depth $\tau_{\Delta \lambda}(r, \varphi)$, from which the relative intensity at location $(r, \varphi)$ is calculated:

$I_{\Delta \lambda}(r, \varphi)=\exp \left(-\left(\tau_{\Delta \lambda}(r, \varphi)\right) \quad(r \geq R)\right.$.

This intensity distribution, affected by the exosphere, and the un-affected solar intensity

$\begin{aligned} I_{\Delta \lambda}^{\odot}(r, \varphi) & \equiv 1 & & r \geq R \\ & \equiv 0 & & r<R\end{aligned}$

are folded with a scattering function $\sigma(r)$, which we have constructed by a weighted sum of two Gaussians and a flat distribution such that the observed distribution of stray light inside Mercury's disk could be reproduced. The resulting calculated intensity difference

$\Delta I_{\Delta \lambda}^{\text {calc }}(r, \varphi)=\left(I_{\Delta \lambda}^{\odot}(r, \varphi)-I_{\Delta \lambda}(r, \varphi)\right) \otimes \sigma(r)$ and the corresponding equivalent widths

$W_{\lambda}^{\text {calc }}=\int_{-\infty}^{+\infty} \Delta I_{\Delta \lambda}^{\text {calc }}(r, \varphi) \mathrm{d} \lambda$

are compared with the observed values. The free parameters $n_{0}$ and $H$ are chosen such to give a reasonable fit. For the Doppler width $\Delta \lambda_{\mathrm{D}}$ we set $=3.1 \mathrm{pm}$, as derived from the observed wavelength shape of the absorption excess (Fig. 3). There is some ambiguity in the choice of $n_{0}$ and $\mathrm{H}$ in the sense that a lower value for $\mathrm{H}$ can be compensated by a higher value for $n_{0}$. Figure 5 shows the height dependence of observed equivalence widths together with that of synthetic ones for several combinations of $H$ and $n_{0}$. The results of this analysis are given in Table 2 for four locations along Mercury's limb.

\section{Discussion}

In this paper, we report on the first successful attempt to detect the sodium exosphere of Mercury by a line absorption effect 
outside the planet's limb. These observations are in several aspects complementary to the observations of line emission at the sun-illuminated side part of the disk. The emission displays the two-dimensional distribution of the exospheric sodium, with the height dimension being projected. In our case, we directly see the height distribution of the absorbing matter, restricted to the planet's terminator, however. The observed emission in the $\mathrm{Na} \mathrm{D}$ lines is mainly produced by resonance scattering, with some contribution due to cascading following recombination of sodium ions, the latter depending on the concentration of the sodium ions. The absorption detected by us depends only on the number of neutral sodium atoms at ground state along the line-of-sight. Unfortunately, transits are rare events (next will be 2006-Nov.-08). Stellar occultations can provide additional absorption determinations, restricted, however, at two opposite azimuthal positions. In view of the non-uniform distribution of the exospheric matter, occultations may often fail to yield a positive absorption signal.

The observed concentration of the sodium exosphere above the polar caps is a strong hint that the dipole-like magnetic field of Mercury plays an important rôle for the formation of the exosphere, either by directing the eroding solar wind towards the polar regions (Ip \& Kopp 2002) or, more indirectly, by influencing transport mechanisms of sodium, leading to an enhancement at the polar surfaces. Polar concentrations have also frequently been observed in line emission (Potter \& Morgan 1990; Sprague et al. 1997, e.g.). Along the western planetary limb (the sun rising terminator), we find a sodium density $\approx 40 \%$ of that above the poles, but no significant absorption could be detected along the eastern planetary limb (the sun setting terminator). Differences between morning - and evening terminator of the same sense were also found by Sprague et al. (1997) from observations of line emission between 1985-1988. Several theoretical investigations on transport mechanisms (Ip 1990, 1993; Sprague 1992) predict enhancement of re-impacting sodium at the night-side surface of Mercury, with subsequent "evaporation" at sun rise.

For the column densities of neutral sodium above the polar caps we find $\left(\approx 3 \times 10^{10} \mathrm{~cm}^{-2}\right)$. This is lower than typical values of several $10^{11} \mathrm{~cm}^{-2}$ derived from emission line spectroscopy, but are within the range found by Sprague et al. (1997). During the days before the Mercury transit, the solar activity was at a relative low level (relative sunspot numbers 65-93), and no geoeffective coronal mass ejections were reported; a huge coronal hole existed at the southern hemisphere producing a high speed wind. Observations of the line emission at the sun illuminated part of Mercury's disk, although strongly influenced by seeing, indicate considerable variability of the distribution and strength of the emission (Potter et al. 1999; Killen et al. 2001). The exosphere of Mercury seems to be far from being static and each individual observation gives only a snapshot of its physical state.

The Doppler width of the excess absorption profile corresponds to a rms velocity of $1.6 \mathrm{~km} \mathrm{~s}^{-1}$ along the line-of-sight.
If completely of thermal origin, a kinetic temperature of the sodium atoms would be $3540 \mathrm{~K}$, which is much larger than the $500 \mathrm{~K}$ derived from the width of emission lines (Potter \& Morgan 1985). Assuming $T_{k}=500 \mathrm{~K}$, a non-thermal rms velocity of $1 \mathrm{~km} \mathrm{~s}^{-1}$ along the line-of-sight would explain the observed width of the absorption feature.

The transit of a planet in front of its central star not only blocks the integral stellar light in proportion to the occulting area of its disk but also has a spectral signature in the strong resonance lines, analogous to what we have seen during the recent Mercury transit. Since spectral photometry, being a relative measurement, can in principle be achieved with a much higher sensitivity than absolute photometry, monitoring stars for such spectral changes is a tool to study exosolar planets. Integrating the profile differences in an annulus around Mercury and multiplying the result with the area ratio of the sampling annulus to that of the solar disk (from a view point at infinity) we find that at the central wavelength of the Mercury absorption feature the disk-integrated solar $\mathrm{Na}_{2}$ profile would show an additional absorption dip of $10^{-6}$ of the undisturbed intensity at this profile point.

Acknowledgements. The observations were done with the VTT on Tenerife, being operated by the Kiepenheuer-Institut für Sonnenphysik (Freiburg) at the Observatorio del Teide of the Instituto de Astrofísica de Canarias. We thank Horst Balthasar (Astrophysikalisches Institut Potsdam) for helpful discussions and the referee for comments leading to improvements of the paper.

\section{References}

Bida, T. A., Killen, R. M., \& Morgan, T. H. 2000, Nature, 404, 159 Bradfoot, A. L., Shemansky, D. E., \& Kumar, S. 1976, Geophys. Res. Lett., 3, 577

Ip, W.-H. 1990, ApJ, 356, 675

Ip, W.-H. 1993, ApJ, 418, 451

Ip, W.-H., \& Kopp, A. 2002, J. Geophys. Res., 107, 1348

Kentischer, T., Schmidt, W., Sigwarth, M., \& v. Uexküll, M. 1998, A\&A, 340, 569

Killen, R. M., Potter, A. E., \& Morgan, T. H. 1990, Icarus, 85, 145

Killen, R. M., Potter, A. E., Reiff, P., et al. 2001, J. Geophys. Res., 106, 20509

Lammer, H., Wurz, P., Patel, M. R., et al. 2003, Icarus, 166, 238

Potter, A. E., \& Morgan, T. H. 1985, Science, 229, 651

Potter, A. E., \& Morgan, T. H. 1986, Icarus, 67, 336

Potter, A. E., \& Morgan, T. H. 1990, Science, 248, 835

Potter, A. E., Killen, R. M., \& Morgan, T. H. 1999, Planetary and Space Science, 47, 1441

Schröter, E. H., Soltau, D., \& Wiehr, E. 1985, Vistas in Astronomy, 28,519

Sprague, A. L. 1992, J. Geophys. Res., 97, 18257

Sprague, A. L., Kozlowski, R. W. H., Hunten, D. M., et al. 1997, Icarus, 129, 506

Soltau, D., Berkefeld, T., v.d. Lühe, O., Wöger, F., \& Schelenz, T. 2002, Astron. Nachr., 323, 236

Tritschler, A., Schmidt, W., Langhans, K., \& Kentischer, T. 2002, Sol. Phys., 211, 17 